\title{
A Note on the Phillips Spectral Framework for Ocean Whitecaps*
}

\author{
MiCHAEL L. BANNER \\ School of Mathematics and Statistics, University of New South Wales, Sydney, New South Wales, Australia, \\ and Ocean and Climate Physics Division, Lamont-Doherty Earth Observatory, Columbia University, \\ Palisades, New York \\ CHRISTOPHER J. ZAPPA \\ Ocean and Climate Physics Division, Lamont-Doherty Earth Observatory, Columbia University, \\ Palisades, New York \\ JOHANNES R. GEMMRICH \\ Physics and Astronomy, University of Victoria, Victoria, British Columbia, Canada
}

(Manuscript received 11 June 2013, in final form 14 March 2014)

\begin{abstract}
There has been a recent upsurge in interest in quantifying kinematic, dynamic, and energetic properties of wave breaking in the open ocean, especially in severe sea states. The underpinning observational and modeling framework is provided by the seminal paper of O. M. Phillips. In this note, a fundamental issue contributing to the scatter in results between investigators is highlighted. This issue relates to the choice of the independent variable used in the expression for the spectral density of the mean breaking crest length per unit area. This note investigates the consequences of the different choices of independent variable presently used by various investigators for validating Phillips model predictions for the spectral density of the breaking crest length per unit area and the associated spectral breaking strength coefficient. These spectral measures have a central role in inferring the associated turbulent kinetic energy dissipation rate and the momentum flux to the upper ocean from breaking wave observations.
\end{abstract}

\section{Background}

The capability of imaging breaking waves from airborne (Kleiss and Melville 2010) and stable platforms (Gemmrich et al. 2008; Gemmrich et al. 2013; Sutherland and Melville 2013; Thomson et al. 2009; Zappa et al. 2012) provides exciting prospects for remotely sensing a number of key air-sea interaction processes in the open ocean. These include momentum exchange associated with airflow separation (Mueller and Veron 2009a; Reul et al. 2008; Veron et al. 2007), sea spray generation (de Leeuw

\footnotetext{
* Lamont-Doherty Earth Observatory Contribution Number 7792 .

Corresponding author address: Christopher J. Zappa, Ocean and Climate Physics Division, Lamont-Doherty Earth Observatory, Columbia University, 204E Oceanography, 61 RT 9W, P.O. Box 1000, Palisades, NY 10964.

E-mail: zappa@ldeo.columbia.edu
}

et al. 2011; Mueller and Veron 2009b), enhanced gas exchange (Asher and Wanninkhof 1998a,b; Keeling 1993; Merlivat and Mémery 1983; Woolf 1993, 2005), satellite microwave remote sensing (Anguelova and Webster 2006; Hwang 2012; Hwang et al. 2008; Reul and Chapron 2003), and near-surface and upper-ocean optical variability (Dickey et al. 2011, 2012), among others. There is also a pressing need to include accurate wavebreaking predictions in routine sea state forecasts, which would benefit maritime safety and operations. Realizing this goal depends on optimally transforming information from observed sea surface signatures of breaking waves into spectral wave-breaking momentum flux and dissipation rate source terms.

Historically, Phillips (1985, hereafter P85) proposed a framework, amenable to both measurement and modeling, for quantifying breaking wave properties spectrally. He noted the following: "There is clearly some association of the breaking events with waves of different scales, but it is difficult to make the association in an 
unambiguous way if we consider only the surface configuration at one given instant. A breaking crest may indeed be a local maximum in the instantaneous surface configuration but there is no guarantee that a local wavelength of the breaking wave can be defined clearly. It seems more satisfactory to use the velocity $\mathbf{c}$ of the breaking front as a measure of the scale of the breaking, since this is a well-defined quantity that might (conceptually at any rate) be measured from ciné images of the sea surface" (P85, p. 526).

Phillips introduced the concept of the spectral density of breaking crest length per unit area of sea surface. He proposed (P85, p. 527) to "identify the scales of waves that are breaking by the speeds with which their fronts advance. For the larger-scale breaking events, i.e., those whose phase speeds $c>(2 \pi s) C$, where $C$ is the phase speed of the dominant wave" and $s$ is the significant slope (Huang et al. 1981), "the associated wavenumber is simply $k=g / c^{2},(\mathrm{P} 85$, p. 527). Here, $g$ is the acceleration due to gravity. He also noted that the speed of advance of smaller-breaking events is influenced strongly by long-wave advection, which introduces a substantial complication in the transformation and chose to concentrate on the "larger-scale breaking events." Phillips then proceeded to define the spectral distribution $\Lambda(\mathbf{c})$ such that $\Lambda(\mathbf{c})$ dc is the average total length per unit sea surface area of breaking fronts that have velocities in the range from $\mathbf{c}$ to $\mathbf{c}+\mathbf{d c}$.

This formulation of the $\Lambda(\mathbf{c})$ distribution provides a valuable scale-dependent measure of wave-breaking kinematics and is a potentially very useful diagnostic for understanding and monitoring air-sea interaction. It can be measured remotely using visible (e.g., Kleiss and Melville 2010; Gemmrich et al. 2013; among several others) or infrared (e.g., Sutherland and Melville 2013) imaging from towers or aircraft. In particular, if the knowledge from this growing database of field and laboratory wave-breaking observations can be incorporated robustly into spectral wave forecast models, this database will provide an important tool for both refining and validating model forecasts of air-sea interfacial quantities, including breaking wave energy dissipation rates, momentum fluxes to the currents, and air-sea fluxes of gases and aerosols.

\section{Key aspects of the Phillips $\Lambda(c)$ framework}

According to our understanding, underpinning the P85 breaking wave framework are the assumptions that (i) the velocity of a breaking front is equal to the phase velocity $\mathbf{c}$ of the underlying gravity wave that is breaking; and (ii) the deep-water dispersion formula relates the observed breaker-front velocity to the underlying wavenumber $\mathbf{k}$ of the breaking wave. This implies that Doppler-shifting effects should be taken into account in transforming between the $\mathbf{c}$ and $\mathbf{k}$ domains. Published studies (e.g., Kleiss and Melville 2010; Gemmrich et al. 2013) indicate that Doppler correction produces only modest changes to $\Lambda(\mathbf{c})$ spectra. We also note that the linear deep-water gravity wave dispersion relation underpins (6.3) of P85 to establish the spectral form of the dissipation rate distribution:

$$
\varepsilon(\mathbf{c}) \mathbf{d c}=b(\mathbf{c}) g^{-1} c^{5} \Lambda(\mathbf{c}) \mathbf{d c},
$$

which is associated with wave breaking, expressed in terms of the breaking wave phase velocity c. Here, the coefficient $b$ is the scale-dependent breaking strength. To focus attention on the key issue of the most appropriate independent variable, we define $\mathbf{c}_{b}$ as the "characteristic" velocity of the breaker front (a turbulent layer flow) to distinguish it from the phase velocity $\mathbf{c}$ of the underlying gravity wave that is breaking.

As explained in P85, underpinning (1) is the assumed scaling of the breaker layer cross-sectional area on the wavelength of the breaking wave. This provides a $c^{4}$ contribution through the deep-water gravity wave dispersion relation, under the assumption that the velocity of a given whitecap (i.e., $\mathbf{c}_{b}$ ) scales with the phase velocity (i.e., c) of the underlying breaking wave. The remaining $c^{1}$ dependence arises from the rate of working of the whitecap mass per unit width that is assumed to proceed at velocity $\mathbf{c}$. Thus, the key relationship (1) from P85 depends heavily on invoking the linear gravity wave dispersion relation.

Under assumptions (i) and (ii), linking (1) to his proposed equilibrium range for the spectral wavebreaking dissipation rate in P85, Phillips deduced $\Lambda(\mathbf{k})$ or its equivalent form $\Lambda(\mathbf{c})$ [(6.7) in P85]. The directionally integrated form of $\Lambda(\mathbf{c})$, within the equilibrium subrange is

$$
\Lambda(c)=\chi b^{-1} u_{*}^{3} c^{-6}
$$

where $\chi$ is a proportionality constant and $u_{*}$ is the wind friction velocity. P85 does not specify bounds for the equilibrium range, but Kleiss and Melville (2010) indicate that after converting from the wavenumber to phase speed, the equilibrium range occurs for $u_{*}<c<$ $0.7 c_{p}$, where $c_{p}$ is the speed of the spectral peak waves. To date, measurements of $\Lambda(c)$ have not provided definite support for (2), because within datasets, departures from (2) have been reported, primarily in terms of the magnitude of the power-law exponent of $c$ in the equilibrium range and the location of the latter within the measured $\Lambda(c)$ spectrum. Less attention has been directed 
at the exponent of the wind friction velocity. Overall, between datasets, significant differences are apparent in the reported $\Lambda(c)$ distributions. In an effort to better understand and hopefully reduce this scatter, we revisit the impact of a key aspect of different processing strategies, namely, the composition of $\Lambda(c)$ from breaker video image analyses.

Our primary focus here is on the issue of consistency with requirements (i) and (ii) above in validating the P85 breaking framework. This is achievable in principle by defining $\mathbf{c}$ as the representative initial $\mathbf{c}_{b}$. Aside from conforming to the proposed P85 framework in order to conduct a self-consistent validation of its predictions, there is no uniqueness implied for this choice of $\mathbf{c}$. Other plausible choices of assigning $\mathbf{c}$ to breaking wave data are available, as discussed below.

\section{Current interpretations of the Phillips $\Lambda\left(\mathbf{c}_{b}\right)$ framework}

From the above discussion, it appears clear that P85 chose the fixed, initial breaker-front reference velocity $\mathbf{c}_{b}$ to characterize the scale of each breaking event and to assume the role of the independent variable in his spectral breaking framework. Using $\mathbf{c}_{b}$ as the basis for processing the breaking image data is referred to hereafter as the initial velocity method (IVM). It underlies the $\Lambda(c)$ results reported by Gemmrich et al. (2008, 2013).

Alternative strategies that have been implemented for composing $\Lambda(c)$ account explicitly for the appreciable change in speed of a whitecap after its initiation. In hindsight, this has actually been the preferred practice in previous studies concerned with validating and refining the P85 framework. Statistically, the mean speed of the breaking fronts of a whitecap has been observed to decrease to about half its initial mean speed during its lifetime (e.g., Kleiss and Melville 2011), with associated changes in its crest width and swept area. We note that this slowdown in the breaking front speed is not referred to in the P85 prescription of kinematic variables. Other investigators (e.g., Melville and Matusov 2002; Kleiss and Melville 2010; Jessup and Phadnis 2005; Thomson and Jessup 2009) have considered that this aspect of the P85 framework may be too restrictive and that using a time-dependent breaking front velocity $\mathbf{c}_{b}$ may provide a more fundamental scientific perspective on this problem.

Thus, several variants have developed for ascribing a velocity characterization to breaking fronts. These assign a different $\mathbf{c}_{b}$ value at each time step for each individual breaker crest length determination (Kleiss and Melville 2010, 2011). Under this prescription, within any specific $\mathbf{c}_{b}$ bin of the breaking crest length distribution, there are contributions from short breakers at early stages of their breaking together with contributions from older, longer breakers that have decelerated appreciably. This class of method will be referred to hereafter as the variable velocity method (VVM). However, it should be noted that while this approach utilizes more kinematic breaking front information during active breaking, it still does not capture all the details needed to quantify the dissipation rate. The local thickness, downslope extent, and velocity of the breaker mass relative to the underlying wave orbital motion are missing. These quantities still need to be included as a modified breaking strength coefficient $b^{\prime}$. However, this method involves the explicit loss of information about the scale of the individual waves that are breaking, and hence the $b^{\prime}$ is intrinsically multiscale. Upon further inspection, it is composed of a range of older, larger breakers in their latter, weaker stage, with younger, more vigorous smaller-scale breakers. This would seem to dilute the concept of a breaking strength. The allied shortcoming of the VVM methodology is that it obscures the transformation from $\mathbf{c}$ to $\mathbf{k}$ space, as the speed of the slowing breaker front (a turbulent shear layer, not a wave field) has no established connection to the wavenumber domain through the deep-water wave dispersion relation. Yet the dispersion relation is routinely invoked in spectral wave forecasting applications when attempting to integrate wave-breaking observational data made in $\mathbf{c}_{b}$ space into the radiative transfer equation, which operates in $k$ space (Banner and Morison 2010; Romero et al. 2012). For both methodologies (IVM and VVM), the dispersion relation is used to transform $\Lambda\left(\mathbf{c}_{b}\right)$ data for input to the wave model. Its use for VVMderived data raises significant concerns.

Another method, based on Fourier domain analysis, is performed in the spectral domain, with a marked gain in processing time (Thomson and Jessup 2009; Thomson et al. 2009). For each speed bin, it is believed to calculate a mean breaker length at the mean speed of the breaker during its evolution history. Similar to the VVM, it appears that the Fourier spectral method also reshapes $\Lambda\left(\mathbf{c}_{b}\right)$ relative to the IVM result, decreasing the spectral levels for large $\mathbf{c}$ and boosting the spectral level for small $\mathbf{c}$. Thus, there will be an overall shift in $\mathbf{c}$ toward shorter scales. To present the results from the Fourier method according to the P85 framework, a significant effort is needed to recover the initial breaker velocities from the mean breaker velocities. There are also issues of broadened spectral bandwidth due to this processing methodology that need to be resolved to ensure that higher-order moments converge when integrated. Efforts in this direction have been made in appendix A of Schwendeman et al. (2014).

While these alternate definitions of $\mathbf{c}_{b}$ provide plausible prescriptions for a breaking crest spectral density 
distribution $\Lambda\left(\mathbf{c}_{b}\right)$, they can be shown to produce measurably different results from using the initial $\mathbf{c}_{b}$ prescription proposed by P85. In their detailed methodology paper, Kleiss and Melville (2011) point out that the IVM and VVM methods can produce significant differences (which they refer to as bias) by a factor of $O(175 \%)$ in the first moment and $O(300 \%)$ in the fifth moment of $\Lambda(c)$. This includes a small $(30 \%)$ overestimate of mean spectral levels of $\Lambda\left(c_{b}\right)$ in Gemmrich et al. (2008) from using $L_{\max }$ rather than $L_{\text {mean }}$ for the length of each whitecap event in their IVM processing that has since been rectified (Zappa et al. 2012). From our perspective, it is the VVM methodology that delivers a strong bias away from the P85 framework, which compromises the assessment of its validation.

This note is aimed at drawing attention to this issue. However, we emphasize that there is no implication here that P85 is correct nor that alternative definitions for $\mathbf{c}_{b}$ should not be pursued, we simply argue here that the latter specifications depart measurably from the P85 breaking framework and therefore do not provide a consistent basis for validating its predictions. Potential differences associated with implementing various breaking wave image processing methodologies have already been previously discussed [e.g., Fig. 9 in Kleiss and Melville (2011); Schwendeman et al. 2014]. Here, we provide an additional assessment that quantifies typical differences implicit in these alternative c prescriptions based on using the ellipse method (Gemmrich et al. 2008). We use this to revisit the issue of obtaining an unbiased validation of the P85 breaking wave framework.

\section{Present study interpretation of the Phillips $\Lambda\left(c_{b}\right)$ framework}

Our purpose in this note is to quantify likely differences for $\Lambda(\mathbf{c})$ distributions measured using fixed initial breaker-front velocities (e.g., Gemmrich et al. 2008; Gemmrich et al. 2013) compared with incorporating the breaker-front slowdown explicitly (e.g., Melville and Matusov 2002; Kleiss and Melville 2010). We open our discussion on the choice of independent variable in relation to recent advances in our knowledge of wave breaking.

\section{a. Breaker-front speed}

To measure $\Lambda(\mathbf{c})$ according to initial breaking front speed, a significant challenge is to accurately determine a representative initial velocity for each tracked breaker, for which our methodology is described below. There is a further nontrivial complication that was not known when the P85 framework appeared: observed values of the initial breaker speed appear to be measurably $[O(20 \%)]$ lower than the linear phase speed of the underlying breaking wave [e.g., (Rapp and Melville 1990)]. The factor $\alpha$ that links the initial whitecap speed to the phase speed of the underlying wave is not yet known precisely (Banner et al. 2014), but its magnitude is important for consistency in rescaling measured $\Lambda\left(\mathbf{c}_{b}\right)$ to $\Lambda(\mathbf{c})$ and also when transforming $\Lambda\left(\mathbf{c}_{b}\right)$ to recover $\Lambda(\mathbf{k})$, given the quadratic dependence of $c$ on $k$ in the dispersion relation. However, our present imprecise knowledge of $\alpha$ does not impact on the results presented here, as we are concerned primarily with contrasting the methodologies used to determine $\Lambda\left(\mathbf{c}_{b}\right)$.

\section{b. Spectral changes resulting from breaking}

During the past 2-3 decades, there has also been an ongoing effort to extend the basic understanding of wave breaking in the physical and spectral domains. The recent review article of Perlin et al. (2013) presents a focused account of these advances. Of particular interest is how to extrapolate spectral signatures of breaking laboratory waves in 2D focused, nonlinear wave packets (Meza et al. 2000; Rapp and Melville 1990; Tian et al. 2011) to natural wind waves in the field. The laboratory measurements suggest that breaking is not localized in the spectral sense and thus cannot be linked to a single crest speed. One of the reviewers of this note suggested the slowing breaker-front speed during breaking may be associated with a shift toward increasing wavenumbers in the dissipation source term in the spectral wave energy balance evolution equation. However, it is also possible that the local breaking crest slowdown [see (i)] could be playing a role in this spectral redistribution. Further, there is no consensus on how the observed changes associated with breaking in unidirectional, waveguide-constrained, narrow bandwidth, frequencydomain laboratory findings relate to changes associated with breaking in the much broader bandwidth, directional ocean wave spectra. Given this lack of understanding of the spectral impact of breaking in a broad bandwidth ocean spectrum, a distinct advantage of the IVM approach is that it allows subsequent reshaping, via use of a suitable filter window, of the spectral bandwidth of breaking influence when transforming from $\mathbf{c}$ to $\mathbf{k}$.

\section{Sensitivity of $\Lambda\left(c_{b}\right)$ results to the IVM and VVM approaches}

Recent field measurements of ocean-breaking waves were performed from the Research Platform $(\mathrm{R} / \mathrm{P})$ Floating Instrument Platform (FLIP) in the Santa Barbara Channel (hereafter SBC) and in the southeastern Pacific Ocean near Hawaii (hereafter HI) during the Radiance in a Dynamic Ocean (RaDyO) experiment (Zappa et al. 
2012). The SBC data comprised young seas [twenty-four 40-min records with mean wave age $c_{p} / u_{*}=22.3$ (range: 18.3-25)], with occasional breaking of the dominant wind sea. The breaking waves in the HI data were largely confined above the spectral peak as these wind seas were close to full development [fourteen 40-min records with mean wave age $c_{p} / u_{*}=48.1$ (range: 45 51.7)]. We processed whitecap imagery using the ellipse method (Gemmrich et al. 2008) according to both the IVM and VVM approaches.

Figure 1 compares measured $\Lambda\left(c_{b}\right)$ spectral distributions for the HI (left) and SBC (right) datasets, using both the IVM (black diamonds) and VVM (gray triangles) approaches, as well as their ratios and their corresponding scaled fifth moment $\rho g^{-1} c^{5} \Lambda(c)$, where $\rho$ is the water density. Note that the true dissipation rate would require another scaling factor incorporating a breaking strength coefficient $b$.

It is seen in Figs. 1a and 1b that the VVM approach systematically reduces the $\Lambda\left(c_{b}\right)$ spectral density for the faster-traveling breakers and increases the $\Lambda\left(c_{b}\right)$ spectral density for the slower breakers. It is also seen that the VVM approach necessarily steepens the slope of the falloff in $\Lambda\left(c_{b}\right)$ for the longer, faster-moving breakers and increases $\Lambda\left(c_{b}\right)$ for the slow-moving waves. To illustrate the difference the methodology can make, for the HI data in Fig. 1c, for the subrange $3.75 \leq c_{p} \leq 6.75$, the power-law exponent is $-6.5 \pm 0.75$ for IVM and $-8.0 \pm 1.03$ for VVM, where the uncertainty bounds are $95 \%$ confidence intervals. Also, for these data, the mean differences in the integrated $\Lambda\left(c_{b}\right)$ distributions are less than $\pm 3 \%$. However, the integrated, scaled fifthmoment spectral densities show a factor of 2.2 enhancement for the IVM approach relative to the VVM approach. Finally, we point out that while the average difference over the entire scaled fifth-moment spectrum is only a factor of $O(2)$, for the longest (fastest) waves the difference is a factor of 4 and considerably higher than this for the shortest waves. We note that the differences reported here using the IVM and VVM methods were for our two RaDyO project datasets. Further differences are anticipated depending on the bandwidth of the dataset as determined by the wind speed, wave age, and the resolution capability of the imaging methodology.

\section{Discussion}

Since P85 appeared, scatter in the published results for $\Lambda(c)$ [e.g., Fig. 1 in Kleiss and Melville (2010)], and consequently the integrated breaking strength parameter $b$, has not yet provided confidence in the applicability of the P85 framework to natural-breaking wind waves in the ocean. However, this perception may change with the very recent results of Sutherland and Melville (2013) based on infrared imagery, which have increased the bandwidth of reliable kinematical and geometrical breaking front measurements, extending the resolution to include short-breaking wave scales that need not break with air entrainment. These initial IR stereo results appear to support a more extensive equilibrium range behavior. Nevertheless, uncertainty will persist due to differences in the processing methodology, reinforcing the timeliness of revisiting key issues of the seminal P85 framework using such exciting new data.

There are other key issues specifically concerned with the kinematical aspects of the P85 framework that warrant close scrutiny. The Duncan (1981) scaling that underlies the P85 model prediction of the form of the spectral energy dissipation rate $\varepsilon \sim c^{-5}$ has never been verified in the field. Also, the predicted $\Lambda(c) \sim c^{-6}$ dependence in P85 is only observed over a subset of scales that extend well into the fast $c$ range very close to the spectral peak. This is shifted considerably to faster wave speeds above the nominal equilibrium range $u_{*}<c<$ $0.7 c_{p}$ (Kleiss and Melville 2010) in the $c$ domain. There are also interesting implications for the breaking strength coefficient $b(\mathbf{c})$ in (1)-(2) needed to link the kinematics to the energetics, as discussed in considerable detail by Romero et al. (2012) and Schwendeman et al. (2014), among others. In this context, we point out that the IVM and VVM approaches may also have a significant influence on $b(\mathbf{c})$. For the IVM, $b(\mathbf{c})$ will be constituted from breakers within the same bandwidth, with comparable wave ages. In contrast, for the VVM method, $b(\mathbf{c})$ will be constituted from breakers from a much broader bandwidth, ranging from smaller wavenumbers of relatively older wave ages to higher wavenumbers of younger wave ages. This smearing of the spectral breaking strength in $\mathbf{c}$ space associated with the VVM method appears to reduce the dynamic range of the breaking strength coefficient $b(\mathbf{c})$ irreversibly and thereby diminish its utility. This perceived deficiency is additional to precluding the use of the dispersion relation to convert from $\mathbf{c}$ to $\mathbf{k}$ space described earlier.

Without reconciling the concerns raised in this note, the P85 framework remains hypothetical and its validity uncertain. Clearly, the traditional way forward is to investigate the P85 kinematical framework as it was defined before concluding what aspects need further refinement.

\section{Concluding remarks}

In the ongoing quest to provide unbiased validation of the spectral breaking wave framework proposed by P85, 

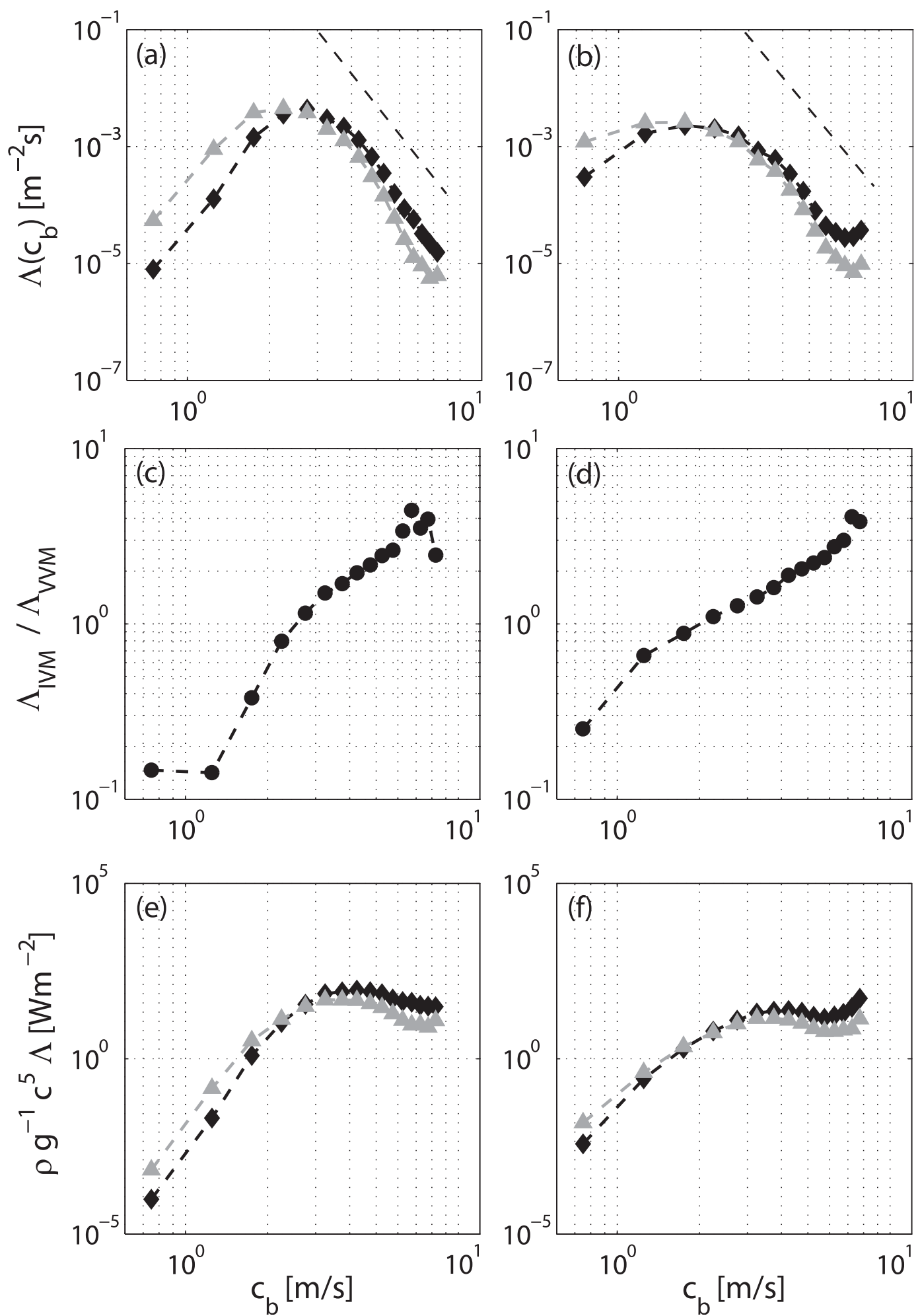

FIG. 1. Spectral distributions of (a) $\Lambda\left(c_{b}\right)$ for the southeastern Pacific, (b) $\Lambda\left(c_{b}\right)$ for the SBC, (c) $\Lambda_{\mathrm{IVM}}\left(c_{b}\right) / \Lambda_{\mathrm{VVM}}\left(c_{b}\right)$ for the southeastern Pacific, (d) $\Lambda_{\mathrm{IVM}}\left(c_{b}\right) / \Lambda_{\mathrm{VVM}}\left(c_{b}\right)$ for the SBC, (e) scaled fifth moment of $\Lambda\left(c_{b}\right)$ for the southeastern Pacific, and (f) scaled fifth moment of $\Lambda\left(c_{b}\right)$ for the SBC. The mean wave age $C / u_{*}$ is 48.1 in the southeastern Pacific with a dominant phase speed $C$ of $13.4 \pm 0.5 \mathrm{~m} \mathrm{~s}^{-1}$. For the SBC, the mean wave age is 22.3 with $C=7.0 \pm$ $1.3 \mathrm{~m} \mathrm{~s}^{-1}$. Black diamonds correspond to the IVM approach, and gray triangles correspond to the VVM approach. Also, the $c_{b}^{-6}$ power-law falloff corresponding to the P85 equilibrium range model for $\Lambda\left(c_{b}\right)$ is shown as a dashed line in (a) and (b). 
we advocate a consolidated effort in the data processing strategies that conforms to the underlying assumptions of the framework, at least until the applicability or otherwise of this framework has been determined. Specifically, using the initial breaking front velocity to characterize each breaker is an explicit requirement for that framework.

We have shown that departing from the P85 spectral framework by using a time-dependent breaker-front speed instead of the fixed initial breaking front speed redistributes the $\Lambda(c)$ distribution to slower waves. For our RaDyO datasets, the integrated fifth moment of $\Lambda(c)$ is reduced by a factor of 2.2 and its local fifth moment for these faster scales is reduced by up to a factor of 4 and up to a factor of 10 for slow-moving wave scales. For the Hawaii dataset, we also found that the fitted power-law exponent for the $\Lambda(c)$ subrange toward the faster-moving waves is reduced appreciably, indicatively to -8 for the time-dependent $\Lambda$ composition compared with -6.5 from the initial speed $\Lambda$ composition. The consequences of these differences in measured $\Lambda(c)$ distributions highlighted here impact on the allied issue of the associated spectral breaking strength coefficient $b(\mathbf{c})$ needed to relate the spectral kinematic breaking front measurements to spectral breaking dissipation rates. Irrespective of whether one is targeting $b(\mathbf{c})$ or an integrated value $b_{\text {eff }}$, the above differences found for $\Lambda(c)$ have a significant corresponding impact on the breaking strength measures. While this note does not aim to resolve which approach is correct, nor place any restriction on alternative definitions, it does highlight the need for uniformity/consistency for evaluating the applicability of the P85 framework. This need is emphasized by the very recent availability of stereo IR breaking front spectral data that show equilibrium range behavior over a significantly broader spectral bandwidth.

Acknowledgments. This work was funded by the Office of Naval Research under the Radiance Dynamics of the Ocean (RaDyO) DRI, with Grant Numbers N00014-06-1-0047 and N00014-11-1-0054 (M. Banner), N00014-06-1-0372 and N00014-11-1-0168 (C. Zappa), and N00014-11-1-0015 (J. Gemmrich). We thank the anonymous reviewers for their helpful comments and recommendations.

\section{REFERENCES}

Anguelova, M. D., and F. Webster, 2006: Whitecap coverage from satellite measurements: A first step toward modeling the variability of oceanic whitecaps. J. Geophys. Res., 111 C03017, doi:10.1029/2005JC003158.

Asher, W. E., and R. Wanninkhof, 1998a: Transient tracers and air-sea gas transfer. J. Geophys. Res., 103, 15939-15958, doi:10.1029/98JC00379.
— and - 1998b: The effect of bubble-mediated gas transfer on purposeful dual-gaseous tracer experiments. J. Geophys. Res., 103, $10555-10560$, doi:10.1029/98JC00245.

Banner, M. L., and R. P. Morison, 2010: Refined source terms in wind wave models with explicit wave breaking forecasts. Part I: Model framework and validation against field data. Ocean Modell., 33, 177-189, doi:10.1016/j.ocemod.2010.01.002.

_ _ X. Barthelemy, F. Fedele, M. Allis, A. Benetazzo, F. Dias, and W. L. Peirson, 2014: Linking reduced breaking crest speeds to unsteady nonlinear water wave group behavior. Phys. Rev. Lett., 112, 114502, doi:10.1103/PhysRevLett.112.114502.

de Leeuw, G., E. L Andreas, M. D. Anguelova, C. W. Fairall, E. R. Lewis, C. O'Dowd, M. Schulz, and S. E. Schwartz, 2011: Production flux of sea spray aerosol. Rev. Geophys., 49, RG2001, doi:10.1029/2010RG000349.

Dickey, T., G. Kattawar, and K. Voss, 2011: Shedding new light on light in the ocean. Phys. Today, 64, 44-50, doi:10.1063/1.3580492. , and Coauthors, 2012: Introduction to special section on recent advances in the study of optical variability in the nearsurface and upper ocean. J. Geophys. Res., 117, C00H20, doi:10.1029/2012JC007964.

Duncan, J. H., 1981: An experimental investigation of breaking waves produced by a towed hydrofoil. Proc. Roy. Soc. London, A377, 331-348, doi:10.1098/rspa.1981.0127.

Gemmrich, J. R., M. L. Banner, and C. Garrett, 2008: Spectrally resolved energy dissipation and momentum flux of breaking waves. J. Phys. Oceanogr., 38, 1296-1312, doi:10.1175/ 2007JPO3762.1.

—, C. J. Zappa, M. L. Banner, and R. P. Morison, 2013: Wave breaking in developing and mature seas. J. Geophys. Res. Oceans, 118, 4542-4552, doi:10.1002/jgrc.20334.

Huang, N. E., S. R. Long, and L. F. Bliven, 1981: On the importance of the significant slope in empirical wind-wave studies. J. Phys. Oceanogr., 11, 569-573, doi:10.1175/1520-0485(1981)011<0569: OTIOTS $>2.0 . \mathrm{CO} ; 2$.

Hwang, P. A., 2012: Foam and roughness effects on passive microwave remote sensing of the ocean. IEEE Trans. Geosci. Remote Sens., 50, 2978-2985, doi:10.1109/TGRS.2011.2177666.

_ M. A. Sletten, and J. V. Toporkov, 2008: Analysis of radar sea return for breaking wave investigation. J. Geophys. Res., 113, C02003, doi:10.1029/2007JC004319.

Jessup, A. T., and K. R. Phadnis, 2005: Measurement of the geometric and kinematic properties of microscale breaking waves from infrared imagery using a PIV algorithm. Meas. Sci. Technol., 16, 1961-1969, doi:10.1088/0957-0233/16/10/011.

Keeling, R. F., 1993: On the role of large bubbles in air-sea gas exchange and supersaturation in the ocean. J. Mar. Res., 51, 237-271, doi:10.1357/0022240933223800.

Kleiss, J. M., and W. K. Melville, 2010: Observations of wave breaking kinematics in fetch-limited seas. J. Phys. Oceanogr., 40, 2575-2604, doi:10.1175/2010JPO4383.1.

$\longrightarrow$, and - 2011: The analysis of sea surface imagery for white cap kinematics. J. Atmos. Oceanic Technol., 28, 219-243, doi:10.1175/2010JTECHO744.1.

Melville, W. K., and P. Matusov, 2002: Distribution of breaking waves at the ocean surface. Nature, 417, 58-63, doi:10.1038/ 417058a.

Merlivat, L., and L. Mémery, 1983: Gas exchange across an airwater interface: Experimental results and modeling of bubble contribution to transfer. J. Geophys. Res., 88, 707-724, doi:10.1029/JC088iC01p00707.

Meza, E., J. Zhang, and R. Seymour, 2000: Free-wave energy dissipation in experimental breaking waves. J. Phys. Oceanogr., 
30, 2404-2418, doi:10.1175/1520-0485(2000)030<2404: FWEDIE $>2.0$. CO;2.

Mueller, J., and F. Veron, 2009a: A nonlinear formulation of the bulk surface stress over the ocean through a simple feedback mechanism. Bound.-Layer Meteor., 130, 117-134, doi:10.1007/ s10546-008-9334-6.

— function. J. Phys. Oceanogr., 39, 2363-2372, doi:10.1175/ 2009JPO4113.1.

Perlin, M., W. Choi, and Z. Tian, 2013: Breaking waves in deep and intermediate waters. Annu. Rev. Fluid Mech., 45, 115-145, doi:10.1146/annurev-fluid-011212-140721.

Phillips, O. M., 1985: Spectral and statistical properties of the equilibrium range in wind-generated gravity waves. J. Fluid Mech., 156, 505-531, doi:10.1017/S0022112085002221.

Rapp, R. J., and W. K. Melville, 1990: Laboratory measurements of deep-water breaking waves. Philos. Trans. Roy. Soc. London, A331, 735-800, doi:10.1098/rsta.1990.0098.

Reul, N., and B. Chapron, 2003: A model of sea-foam thickness distribution for passive microwave remote sensing applications. J. Geophys. Res., 108, 3321, doi:10.1029/ $2003 \mathrm{JC} 001887$.

- H. Branger, and J.-P. Giovanangeli, 2008: Air flow structure over short-gravity breaking water waves. Bound.-Layer Meteor., 126, 477-505, doi:10.1007/s10546-007-9240-3.

Romero, L., W. K. Melville, and J. Kleiss, 2012: Spectral energy dissipation due to surface wave breaking. J. Phys. Oceanogr., 42, 1421-1444, doi:10.1175/JPO-D-11-072.1.
Schwendeman, M., J. Thomson, and J. R. Gemmrich, 2014: Wave breaking dissipation in a young wind sea. J. Phys. Oceanogr., 44, 104-127, doi:10.1175/JPO-D-12-0237.1.

Sutherland, P., and W. K. Melville, 2013: Field measurements and scaling of ocean surface wave-breaking statistics. Geophys. Res. Lett., 40, 3074-3079, doi:10.1002/grl.50584.

Thomson, J., and A. Jessup, 2009: A Fourier-based method for the distribution of breaking crests from video observations. J. Atmos. Oceanic Technol., 26, 1663-1671, doi:10.1175/ 2009JTECHO622.1.

_ J. R. Gemmrich, and A. T. Jessup, 2009: Energy dissipation and the spectral distribution of whitecaps. Geophys. Res. Lett., 36, L11601, doi:10.1029/2009GL038201.

Tian, Z., M. Perlin, and W. Choi, 2011: Frequency spectra evolution of two-dimensional focusing wave groups in finite depth water. J. Fluid Mech., 688, 169-194, doi:10.1017/jfm.2011.371.

Veron, F., G. Saxena, and S. Misra, 2007: Measurements of viscous tangential stress in the airflow above wind waves. Geophys. Res. Lett., 34, L19603, doi:10.1029/2007GL031242.

Woolf, D. K., 1993: Bubbles and the air-sea transfer velocity of gases. Atmos.-Ocean, 31, 517-540, doi:10.1080/07055900.1993.9649484.

_ 2005: Parameterization of gas transfer velocities and seastate-dependent wave breaking. Tellus, 57B, 87-94, doi:10.1111/ j.1600-0889.2005.00139.x.

Zappa, C. J., M. L. Banner, J. R. Gemmrich, H. Schultz, R. P. Morison, D. A. LeBel, and T. Dickey, 2012: An overview of sea state conditions and air-sea fluxes during $\mathrm{RaDyO}$. J. Geophys. Res., 117, C00H19, doi:10.1029/2011JC007336. 